\title{
DIRECT PRINTING OF SILVER NANO-PARTICLES BASED INK ON POLYDIMETHYLSILOXANE (PDMS) FOR MEMS DEVICES
}

\author{
Yoontae Kim* ${ }^{*}$ Xiang Ren, and Hongseok (Moses) Noh \\ Drexel University, Philadelphia, Pennsylvania, USA
}

\begin{abstract}
We introduce the use of inkjet printing techniques for rapid fabrication of conductive silver microelectrodes on polydimethylsiloxane (PDMS). An extensive experimental characterization study on the effect of printing parameters on the electrode quality is presented here. We also demonstrate the utility of the microelectrode-printing technique by making quadruple electrodes and using for dielectrophoretic (DEP) manipulation of polystyrene microbeads.
\end{abstract}

\section{INTRODUCTION}

Microfabricated PDMS devices are widely used for a variety of applications such as microfluidic components, lab-on-a-chip systems, chemical and biological sensors. These devices often requires integrated micropatterned electrode for electrokinetic manipulation of liquids and particles, and electrical/ electrochemical sensing. Conventional methods to create micropatterned electrodes are based on photolithography and thin-film deposition techniques[1-3]. Such fabrication process is time consuming, cost-ineffective, and often involves harmful chemicals. Alternative methods have been reported including microcontact printing using patterned PDMS stamp[4], direct injection and solidification of the conductive solution into PDMS channels[5]. However, even these methods necessitate photolithography for master mold fabrication.

In recent years, direct inkjet printing of the conductive solution has attracted research community and industries because of its low-cost and mask-free deposition of materials on various substrates, like terephthalate (PET) or polycarbonate (PC)[6-7]. Silver is a good electrode material because of its high electrical conductivity. A number of researchers have studied on direct printing of ink containing silver nanoparticles using an inkjet printing system. However, most of previous studies focused on making drops and continuous tracks on the polymeric substrates with the exception of PDMS. There has been one study on printing conductive silver line on PDMS with UV ozone treatment as a surface modification[8], but the dimension of the printed line was in millimeter scale and no experimental characterization of the process was reported.

In this paper, we report an extensive experimental characterization study on the effect of printing parameters on the electrode quality for silver ink printing on PDMS substrates. PDMS surface was treated with air plasma prior to printing and various conductive silver lines have been printed on the PDMS substrates. The width and thickness of the printed line and their electrical resistance were carefully measured as varying printing parameters, such as drop spacing (DS), platen temperature, nozzle temperatures, sintering temperatures and time durations. We also printed quadruple electrodes and used them for dielectrophoretic (DEP) manipulation of polystyrene microbeads in order to demonstrate the utility of the printed silver electrodes.

\section{EXPERIMENTS}

Silver Ink

A wide variety of inks are commercially available for the DMP-2800 printing system used in this study. Viscosity, surface tension, and particle size were taken into account for the selection of the ink for the present application. We chose silver ink (DGP 40LT-15C, ANP, Korea) that contains $30 \mathrm{wt} \%$ of silver nanoparticles dispersed in Triethylene Glycol Monoethyl Ether (TGME). The physical properties of the ink at $20^{\circ} \mathrm{C}$ were: viscosity $15.4 \mathrm{cps}$, surface tension $40.2 \mathrm{mN} \mathrm{m}^{-1}$, and average particle size 50 $\mathrm{nm}$.
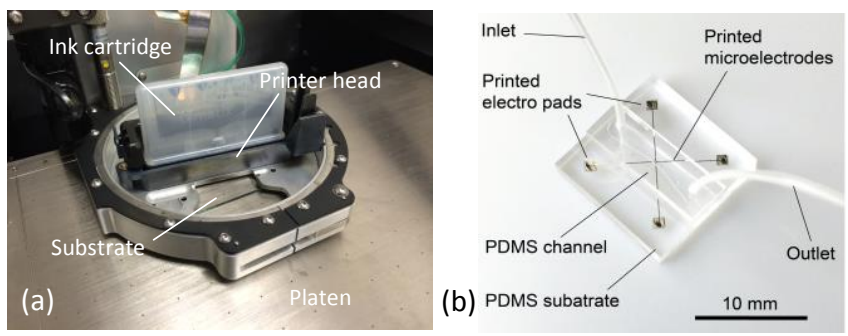

Figure 1: (a) Picture of the inkjet printing system, and (b) printed device used for DEP manipulation of polystyrene microbeads.

\section{Printer Head}

The printer head has 16 nozzles and the nozzle diameter is related to the generated drop size through that nozzle. In this study, we used 1 pL cartridge (DMC-11601, Fujifilm, Japan) which has 9 $\mu \mathrm{m}$ diameter nozzle. One single nozzle was used at a time. Although the piezoelectric print-head can operate at acoustic frequencies in the range of $1-15 \mathrm{kHz}$, it was fixed at $1 \mathrm{kHz}$ for simplicity and thus its influence in drop volume and velocity is ignored[9].

\section{Inkjet Printer and Printing Conditions}

The piezoelectric inkjet printer (Dimatix Material Printer DMP-2800, Fujifilm, Japan) is a software controlled inkjet printer system that allows the user to deposit colloidal fluid on various substrates. This inkjet printing system consists of a printer head, ink cartridges, a platen (substrates holder), two cameras, and Dimatix Drop Manager (DDM) software. Most of printing parameters can be controlled by the user via DDM software.

The printability of fluid highly depends on the fluid characteristics including viscosity and surface tension at jetting temperature. The printer head has a built-in heater, and the fluid is heated up to target jetting temperature. The platen is connected to a vacuum pump to hold thin flexible substrates and this part also has a built-in heater enabling to control substrate temperature, which in turn directly affects evaporation rate of the solvent. There are two cameras on this printing system. The fiducial camera is used to monitor the printed pattern and to measure the pattern sizes. The drop-watcher camera is used to inspect drop formation at the nozzle-air interface. We observed the shape of drops when they are ejected from the nozzles. Moreover, the velocity of ejected drops was monitored by the drop-watcher camera. The nozzle cleanliness also influenced the quality of printed patterns. There are three software-driven methods of cleaning for the nozzle such as purge, blot and spit. These cleaning methods run individually before starting of printing. More printing parameters and detailed printing conditions that we used in this study are presented in Table 1. 
Table 1.Printing process parameters and used printing conditions during the experiments.

\begin{tabular}{|l|l|}
\hline Printing Process Parameters & Predetermined Values \\
\hline Nozzle temperature $\left({ }^{\circ} \mathrm{C}\right)$ & 29 \\
\hline Jetting frequency $(\mathrm{kHz})$ & $1 \mathrm{kHz}$ \\
\hline Jetting velocity $(\mathrm{m} / \mathrm{s})$ & $7-8$ \\
\hline Drop spacing $(\mu \mathrm{m})$ & $5,6,8,10,15,20,25$, and 30 \\
\hline Nozzle height $(\mathrm{mm})$ & 1 \\
\hline $\begin{array}{l}\text { Platen temperature }\left({ }^{\circ} \mathrm{C}\right) \\
(\text { Measured substrate temperature })\end{array}$ & $30(29.2), 40(38.4)$, \\
\hline Sintering temperature $\left({ }^{\circ} \mathrm{C}\right)$ & $90,120,150$, and 180 \\
\hline Sintering time duration $(\min )$ & $15,30,45$, and 60 \\
\hline
\end{tabular}

\section{PDMS Substrate}

Non-uniform substrate thickness can affect printing quality because the distance between nozzle and substrate will change accordingly. In order to minimize the variation in the PDMS substrate thickness, spin coating technique was used. Dow Corning $®$ (Sylgard 184) PDMS are supplied as two-part liquids, a base resin and a curing agent. The base resin and the curing agent were mixed with 10:1 mass ratio, and degassed in a vacuum desiccator for 30 minutes. The mixture was then spin-coated on a soda-lime glass $(2 " \times 2$ ") at $500 \mathrm{rpm}$ for 30 seconds. The PDMS coated soda-lime glass was cured at $80^{\circ} \mathrm{C}$ temperature for 2 hours.

\section{Measurement}

The printed silver lines sintered by a conventional oven under different sintering temperatures and times. After sintering process, the resistance was measured by the 4-wire resistance measurement using a digital multi-meter (2400 SourceMeter ${ }^{\circledR}$, Keithley, USA). The images of printed silver lines were taken by an optical microscope equipped with a CCD camera, so the printed lines had different color map compared to the printing substrate. Matlab can capture each pixel of the images and tell the boundary of the printed lines by a pre-set threshold data. Using this method, we could obtain the average width of the printed lines. In order to get the width of antinodes and nodes, we compared one position's width to its neighbor's width, by setting a confidence interval. The cross-sectional profiles of printed silver lines were obtained using the optical profilometry (NewView 6200, Zygo, USA) before and after sintering.

\section{Quadruple Silver Electrodes Device}

The quadruple silver microelectrodes were printed on PDMS, and then sintered at $180^{\circ} \mathrm{C}$ for 15 minutes. The sintered PDMS part was then attached to a PDMS microchannel $(\mathrm{H}: 100 \mu \mathrm{m} \times \mathrm{W}: 1 \mathrm{~mm} \times$ $\mathrm{L}: 10 \mathrm{~mm}$ ) that was fabricated using replica molding technique with negative photoresist patterns (SU-8 2035, Microchem, USA). The inlet and outlet holes were punched into the channel and silicone tubes were connected to them. Polystyrene microspheres (Polysciences, Inc., USA) with a diameter of $2 \mu \mathrm{m}$ were mixed with deionized water to make a low conductive colloidal mixture for the dielectrophoretic experiments. The measured electrical conductivity of the mixture was $5.68 \times 10^{-5} \mathrm{~S} \mathrm{~m}^{-1}$.

\section{RESUTLS AND DISCUSSION}

\section{Substrate and Surface Treatment}

Direct printing of silver ink on PDMS is challenging because of its elastomeric and hydrophobic nature. There are various techniques to modify the surface properties of PDMS. In this work, PDMS surfaces were modified by an air plasma system
(COVANCE-MP, FEMTO science, Korea). The LF plasma power was $120 \mathrm{~W}$ of $50 \mathrm{~Hz}$ frequency, and the plasma treatment time was 1 $\min$.

Figure 2 shows the dramatic effect of air plasma treatment on printing. The ejected drops evenly spread on plasma-treated PDMS resulting in uniform and continuous silver patterns. In contrast, the ink did not spread, and formed separated on the plasma-untreated PDMS under exactly same printing conditions due to the hydrophobicity of PDMS. The thin silver line created on plasma-treated surface dried quickly while the drops on untreated surface remained as liquid for a long time.
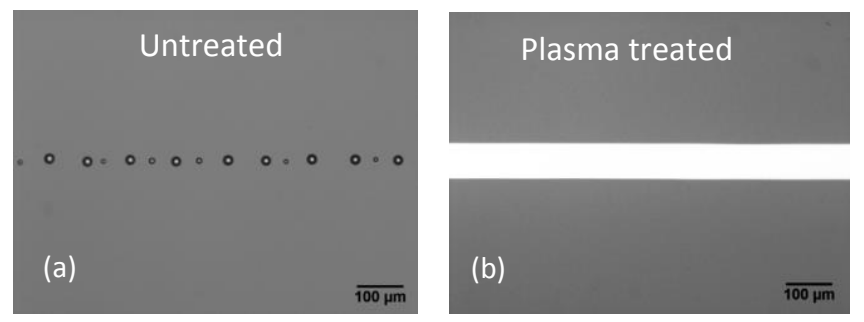

Figure 2: Effect of air plasma treatment on printing (a) Untreated, (b)Plasma treated.

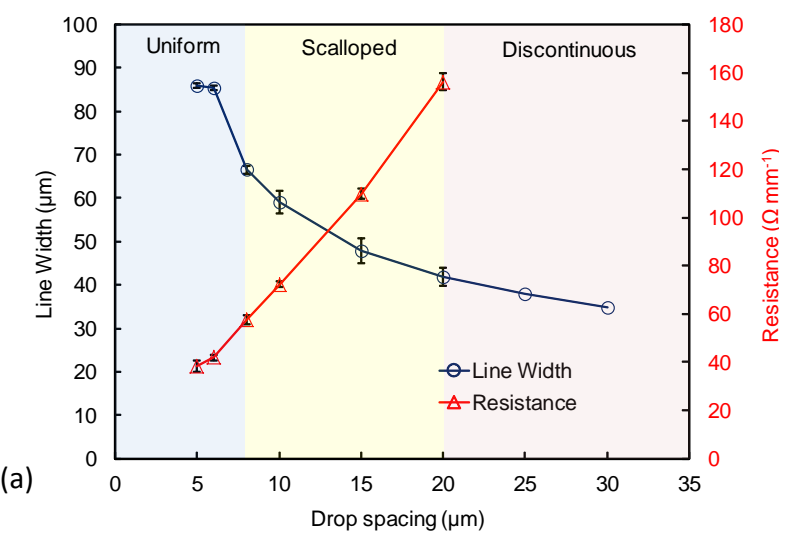

(b)

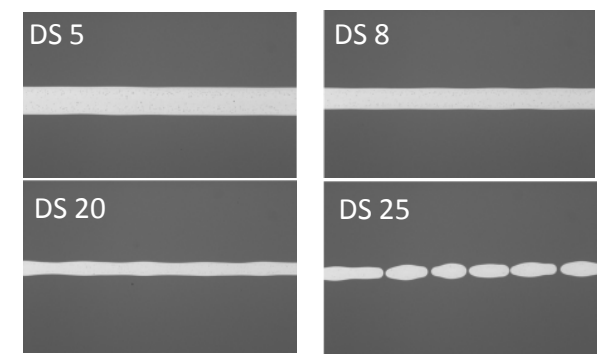

Figure 3: (a) Effect of DS on line width and electrical resistance uniformity, and resistance as function of drop spacing (b) images of printed line with different $D S$.

\section{Drop Spacing}

The meaning of drop spacing (DS) is the center distance between two drops. Figure 3(a) presented that the printed line width highly depends on the DS due to the drop density (drops per inch: dpi) is decided by DS. In this work, we used several different DS, as shown in the table 1 . The maximum drop density was achieved using DS $5 \mu \mathrm{m}$, its resolution was $5080 \mathrm{dpi}$. A pattern printing with DS $30 \mu \mathrm{m}$, the resolution was decreased to $847 \mathrm{dpi}$. Therefore, the printed line width decreases as DS is increased. In addition, the 
electrical resistance of the conductive lines increases. We also observed that relatively straight lines can be produced when DS is below $10 \mu \mathrm{m}$. If DS is higher than $20 \mu \mathrm{m}$, the printed lines were disconnected and the images are shown in figure 3(b).

\section{Substrate Temperature}

We investigated an influence of substrate temperatures on printed silver lines. The platen temperature was changed from 30 ${ }^{\circ} \mathrm{C}$ to $60^{\circ} \mathrm{C}$ in $10^{\circ} \mathrm{C}$ increments. Generally, the surface temperatures of PDMS were slightly lower than platen temperature due to the low thermal conductivities of PDMS and soda-lime glasses. The measured surface temperatures of PDMS were presented in table 1 . The heated substrate promotes the evaporation of ink solvent and prevents line broadening[10]. Figure 4(a) and figure 5 show the effect of substrate temperature. We observed that printed line width decreased with the increase of platen temperature. In contrast, the printed line height gradually increased after increasing platen temperature. As a result, the electrical resistance decreased with line width decrease due to substrate temperature increase because the cross-sectional area actually increased. It means that more silver ink was deposited at higher substrate temperature. It might be due to heat transfer between the substrate and nozzle. If heat was transferred from the platen to the printer head, viscosity and surface tension of silver ink would be decreased while printing. Those physical properties are related to the drop tear off at the nozzle outlet. The ability to withdraw liquid back into the nozzle would be reduced due to decreased surface tension. Therefore, slightly bigger drops can be ejected on PDMS. In practice, the nozzle temperature was slightly increased from $29^{\circ} \mathrm{C}$ to $30.7^{\circ} \mathrm{C}$ while two-line printing. Each line was $13 \mathrm{~mm}$ in length and the platen temperature was 60 ${ }^{\circ} \mathrm{C}$.

(a)
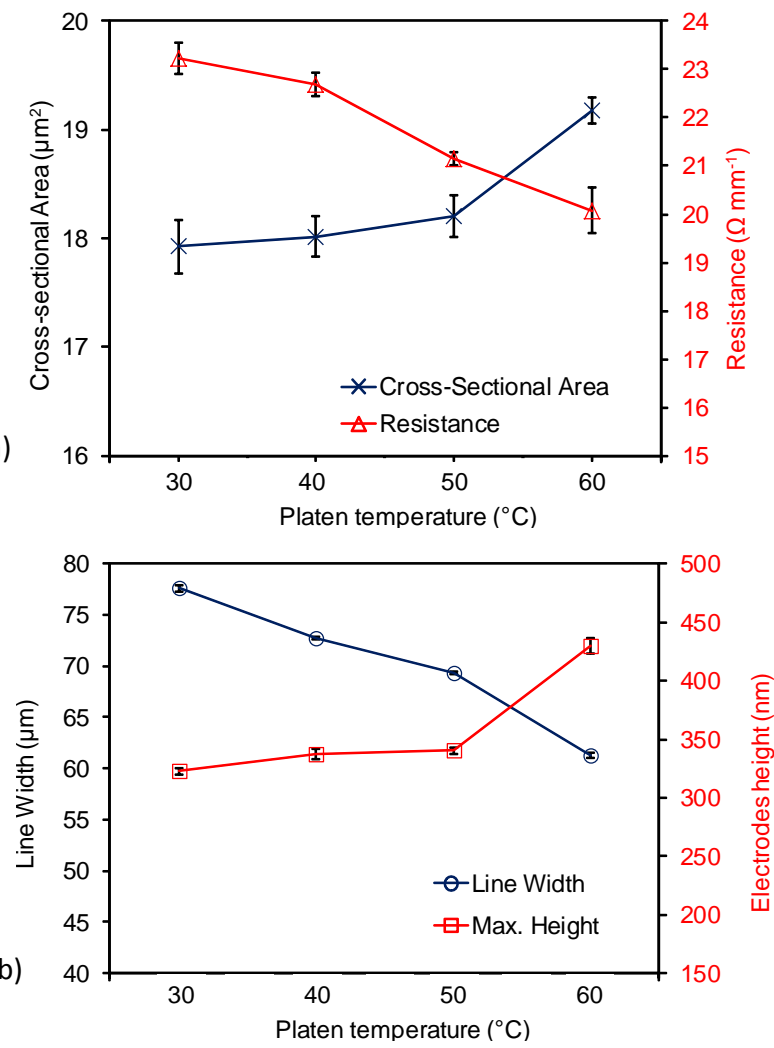

Figure 4: Effect of substrate temperature on (a) line width and height, (b) cross-sectional area and resistance.
We observed the Marangoni effect from the printed silver lines, especially, at side peaks in figure 5(a) and (b). The printed silver lines on PDMS have longer drying time than other materials which have higher thermal conductivities. During the longer period of drying time, the nanoparticles in printed lines can move at the side peaks due to the Marangoni flow[11].

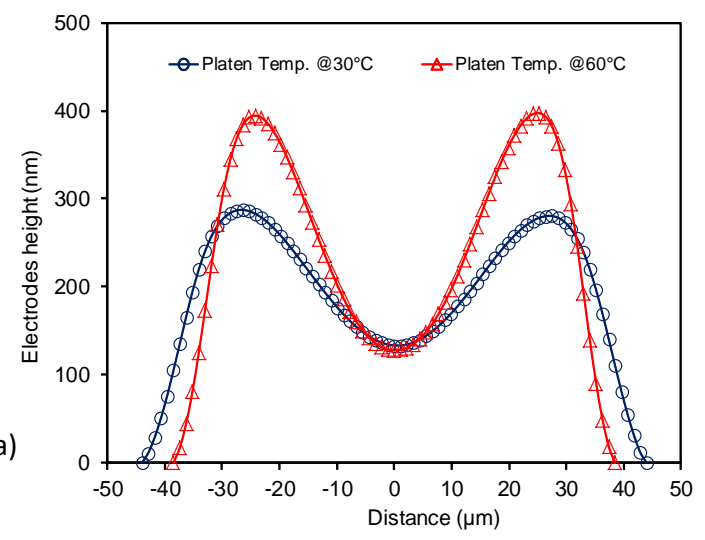

(b)

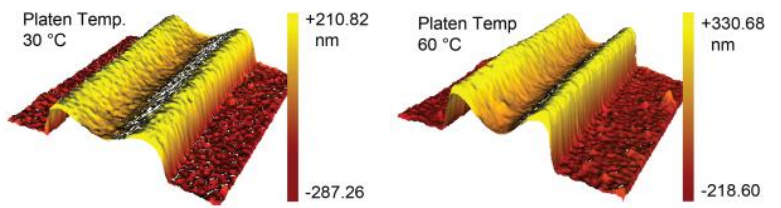

Figure 5: (a) and (b) The cross-sectional profiles of the sintered electrode at the platen temperature of $30^{\circ} \mathrm{C}$ and $60{ }^{\circ} \mathrm{C}$ (obtained from optical profilometry).

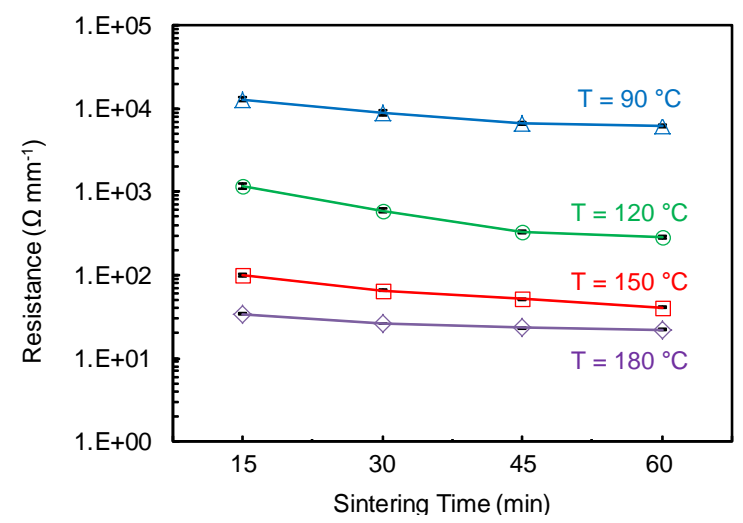

Figure 6: Effect of sintering temperature and time on electrical resistance.

\section{Sintering Temperature}

The printed silver ink must be sintered at high temperature for solvent evaporation and densification. Sintering temperatures and duration times have significant effect on the electrical property of the printed silver electrodes[12]. We printed conductive silver lines and applied four different temperatures for different time durations to investigate the sintering conditions and the results were shown in figure 6 . The electrical resistance of the printed lines decreased significantly with the increase in sintering temperature. The resistance also decreased slightly with sintering time for all different temperatures. We found that the lowest resistance was obtained at $180^{\circ} \mathrm{C}$ for $1 \mathrm{~h}$, and PDMS substrates were not damaged under the 
sintering condition. Note that this sintering temperature is higher than other polymeric substrates such as PET, and $\mathrm{PC}\left(<150^{\circ} \mathrm{C}\right)$.

\section{Dielectrophoretic Experiments}

In order to demonstrate the performance of the printed silver electrodes, we printed quadruple microelectrodes on PDMS and used them for dielectrophoretic manipulation of polystyrene microbeads. The optical microscope with a CCD camera was used to monitor the motions of the particles. A function generator (HP 33120A, HP, USA) was connected to four electrode pads to apply AC electric signals.

Figure 7 (b) and (c) show that particles are collected along the edge of the electrodes. This is indicative of positive DEP at mid frequencies $\left(200 \sim 300 \mathrm{kHz}, 10 \mathrm{~V}_{\mathrm{pp}}\right)$, and the particles move toward area of high electric field strength. At higher frequencies $(700 \mathrm{kHz}$, $10 \mathrm{~V}_{\mathrm{pp}}$ ) particles experience strong negative DEP forces, therefore the particles were pushed away from the edge of the electrodes as shown in figure $7(d)$.
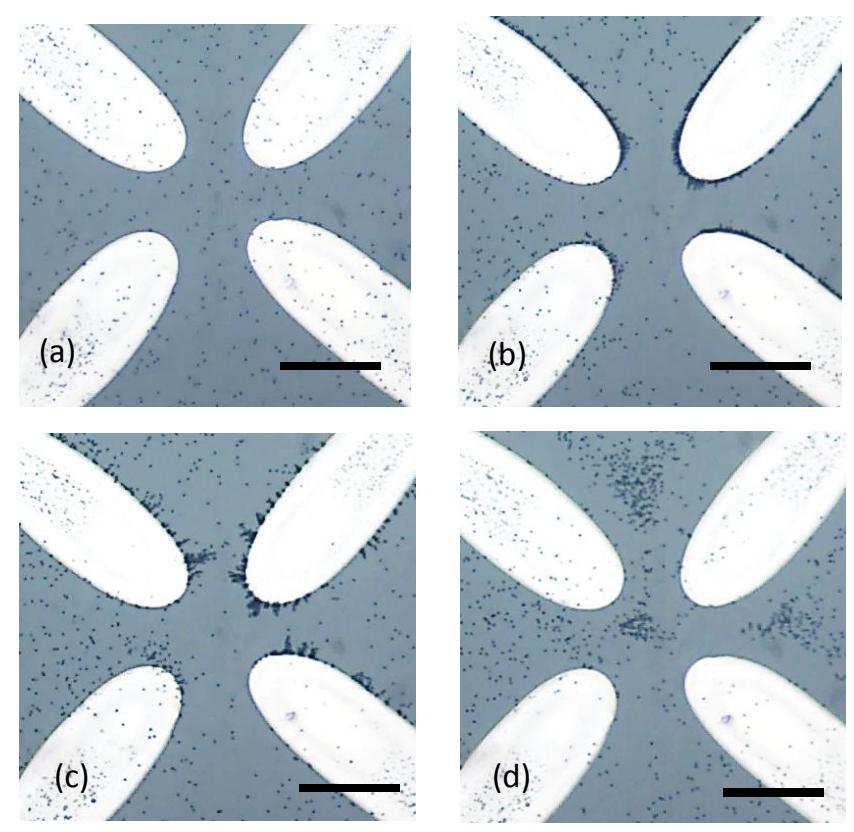

Figure 7: DEP experiment with printed quadruple electrodes: (a) initial random distribution, (b) positive DEP at $200 \mathrm{kHz}, 10 \mathrm{~V}_{p p}$, (c) positive DEP at $300 \mathrm{kHz}, 10 \mathrm{~V}_{p p}$, and (d) negative DEP at $600 \mathrm{kHz}$, $10 V_{p p}$ (scale bar: $\left.100 \mu \mathrm{m}\right)$.

\section{CONCLUSIONS}

We explored direct inkjet printing of silver nanoparticle-based ink on PDMS substrates. Major printing parameters and sintering conditions were thoroughly investigated to identify optimal conditions for electrode fabrication. The findings from the characterization study were then applied to the fabrication of quadruple electrodes for DEP manipulation of microbeads. The direct printing of silver microelectrode on PDMS overcomes many disadvantages of conventional fabrication methods and thus can find many applications.

\section{ACKNOWLEDGEMENTS}

This research was partially supported by the National Science Foundation (Grant No. CBET 0923173, DUE 1022757). Travel support has been generously provided by the Transducer Research Foundation.

\section{REFERENCES}

[1] J.Y. Baek, J.H. An, J.M. Choi, K.S. Park, and S.H. Lee, "Flexible polymeric dry electrodes for the long-term monitoring of ECG," Sensors and Actuators A: Physical, 143, 2 (2008).

[2] S. Chung, J. Lee, H. Song, S. Kim, J. Jeong, and Y. Hong, "Inkjet-printed stretchable silver electrode on wave structured elastomeric substrate," Applied Physics Letters, 98, 15 (2011).

[3] C.Y. Chen, C.L. Chang, T.F. Chien, and C.H. Luo, "Flexible PDMS electrode for one-point wearable wireless bio-potential acquisition," Flexible PDMS electrode for one-point wearable wireless bio-potential acquisition, 203, (2013).

[4] H.L. Gou, J.J. Xu, X.H. Xia, and H.Y. Chen, "Air Plasma Assisting Microcontact Deprinting and Printing for Gold Thin Film and PDMS Patterns", ACS Applied Materials \& Interfaces, 2, 5 (2010).

[5] A.C. Siegel, D.A. Bruzewicz, D.B. Weibel, and G.M. Whitesides, "Microsolidics: Fabrication of Three-Dimensional Metallic Microstructures in Poly(dimethylsiloxane)", Advanced Materials, 19, 5 (2007).

[6] J. Wu, S.L. Hsu, M. Tsai, and W. Hwang, "Inkjet Printing of Low-Temperature Cured Silver Patterns by Using AgNO3/ 1-Dimethylamino-2-propanol Inks on Polymer Substrates," The Journal of Physical Chemistry C, 115, 22 (2011).

[7] T.F. Guo, S.C. Chang, S. Pyo, and Y. Yang, "Vertically Integrated Electronic Circuits via a Combination of Self-Assembled Polyelectrolytes, Ink-Jet Printing, and Electroless Metal Plating Processes," Langmuir 18, 21 (2004).

[8] S. Chung, J. Lee, H. Song, S. Kim, J. Jeong, and Y. Hong, "Inkjet-Printed Stretchable Silver Electrode on Wave Structured Elastomeric Substrate," Applied Physics Letters, 98, 15 (2011).

[9] N. Reis, C. Ainsley, and B. Derby, "Ink-jet Delivery of Particle Suspensions by Piezoelectric Droplet Ejectors," Journal of Applied Physics, 97, 9 (2005).

[10] T.H. van Osch, J. Perelaer, A.W. de Laat, and U.S. Schubert, "Inkjet Printing of Narrow Conductive Tracks on Untreated Polymeric Substrates," Advanced Materials, 20, 2 (2008).

[11] E.L. Talbot, A. Berson, P.S. Brown, and C.D. Bain, "Evaporation of Picoliter Droplets on Surfaces with a Range of Wettabilities and Thermal Conductivities," Physical Review E, 85, 6 (2012).

[12] J.R. Greer, and R.A. Street, "Thermal Cure Effects on Electrical Performance of Nanoparticle Silver Inks," Acta Materialia, 55, 18 (2007).

\section{CONTACT}

*Yoontae Kim, tel: +1-215-895-5820; yk373@drexel.edu 